\title{
Correction
}

Corrigenda to

\section{On the lack of experimental evidence for certain structures in polymers}

Progr Colloid Polym Sci 75:1-3 (1987)

\section{Z. Rigbi}

Technion-Israel Institute of Technology, Haifa, Israel

\begin{abstract}
The direct experimental evidence supporting some of the structures which are postulated to exist in polymers is examined and found lacking, although circumstantial evidence may be strong. An appeal is made for experiments which will develop direct evidence for these sturctures.
\end{abstract}

Key words: Catenates, reptation, model structures, transection.

\section{References}

1. Bunn CW (1939) Trans Faraday Soc 35:482

2. Meyer KH, von Susich G, Valko E (1932) Kolloid-Z 59:208

3. James HM, Guth E (1943) J Chem Phys 11:455

4. Wall JT (1942) J Chem Phys 10:132, 485; idem 11:67, 527

5. Flory PJ, Rehner J (1943) J Chem Phys 11:512

6. Flory PJ, Erman B (1985) Br Polym J 17:96

7. de Gennes PG (1971) J Chem Phys 55:572

8. Doi M, Edwards SF (1978) J Chem Soc Faraday Trans 2 74:1789-1818

9. Graessley WW (1982) Adv Polym Sci 16:1-179
10. Antionetti et al (1986) Macromol 19:793-798

11. Chen Y (1981) J Chem Phys 75:5160

12. Jacobsen H (1984) Macromol 17:705

13. Rigbi Z (1986) J Polym Sci (Phys) 24:443-449

14. Rigbi $Z$ (1980) Adv Polym Sci 36:21-68

Author's address:

Prof. Zvi Rigbi

Technion-Israel Institute of Technology

Haifa, Israel 